\title{
CONVERGENCE ANALYSIS OF PIECEWISE CONTINUOUS COLLOCATION METHODS FOR HIGHER INDEX INTEGRAL ALGEBRAIC EQUATIONS OF THE HESSENBERG TYPE
}

\author{
BABAK SHIRI, SedAghat SHAHMORAD, GHOLAmREZA HOJJATI \\ Faculty of Mathematical Sciences \\ University of Tabriz, 29 Bahmn Boulevard, 5166616471 Tabriz, Iran \\ e-mail: \{shiri, shahmorad, ghojjati\}@tabrizu.ac.ir
}

\begin{abstract}
In this paper, we deal with a system of integral algebraic equations of the Hessenberg type. Using a new index definition, the existence and uniqueness of a solution to this system are studied. The well-known piecewise continuous collocation methods are used to solve this system numerically, and the convergence properties of the perturbed piecewise continuous collocation methods are investigated to obtain the order of convergence for the given numerical methods. Finally, some numerical experiments are provided to support the theoretical results.
\end{abstract}

Keywords: piecewise continuous collocations methods, Volterra integral equations, integral algebraic equations.

\section{Introduction}

Integral Algebraic Equations (IAEs) are not as well known as integral equations or Differential Algebraic Equations (DAEs). But there are some major reasons that make their investigation important. One of these is that a DAE problem can be considered an IAE problem. The second one is that IAEs are more general than integral equations of the first and second kinds and so on.

Here we consider an IAE of the form

$$
\begin{aligned}
A(t) y(t)+\int_{0}^{t} k(t, s) y(s) \mathrm{d} s= & f(t), \\
& t \in I:=[0, T],
\end{aligned}
$$

where $A \in \mathbf{C}\left(I, \mathbb{R}^{r \times r}\right), f \in \mathbf{C}\left(I, \mathbb{R}^{r}\right)$ and $k \in$ $\mathbf{C}\left(D, \mathbb{R}^{r \times r}\right)$ with $D:=\{(t, s): 0 \leq s \leq t \leq T\}$. If $A(t)$ is a nonsingular matrix for all $t \in I$, then multiplying (1) by $A^{-1}$ changes it to a system of Volterra integral equations of the second kind, whose theoretical and numerical analysis has been already investigated (see, e.g., Atkinson, 2001; Hochstadt, 1973; Bandrowski et al., 2010; Saeedi et al., 2011). If $A(t)$ is a singular matrix with constant rank for all $t \in I$, then the system (1) will be an IAE or a singular system of Volterra integral equations of the fourth kind, and if $A(t)$ is a singular matrix with constant rank for some $t \in I$, then the system (1) will be a singular system of Volterra integral equations of the third kind or weakly singular Volterra integral equations.
In this paper, we confine ourselves to a study of integral algebraic equations of the Hessenberg type:

$$
\begin{gathered}
{\left[\begin{array}{cccc}
A_{1,1}(t) & \ldots & A_{1, \nu-1}(t) & 0 \\
\vdots & \ddots & \vdots & \vdots \\
A_{\nu-1,1}(t) & \ldots & 0 & 0 \\
0 & \ldots & 0 & 0
\end{array}\right]\left[\begin{array}{c}
y_{1}(t) \\
y_{2}(t) \\
\vdots \\
y_{\nu}(t)
\end{array}\right]} \\
+\int_{0}^{t}\left[\begin{array}{cccc}
k_{1,1}(t, s) & k_{1,2}(t, s) & \ldots & k_{1, \nu}(t, s) \\
\vdots & \vdots & \ddots & \vdots \\
k_{\nu-1,1}(t, s) & k_{\nu-1,2}(t, s) & \ldots & 0 \\
k_{\nu, 1}(t, s) & 0 & \ldots & 0
\end{array}\right] \\
\times\left[\begin{array}{c}
y_{1}(s) \\
y_{2}(s) \\
\vdots \\
y_{\nu}(s)
\end{array}\right] \mathrm{d}=\left[\begin{array}{c}
f_{1}(t) \\
f_{2}(t) \\
\vdots \\
f_{\nu}(t)
\end{array}\right]
\end{gathered}
$$

where $\prod_{i=1}^{\nu-1} A_{i, \nu-i}$ and $\prod_{i=1}^{\nu} k_{i, \nu+1-i}(t, t)$ are assumed to be invertible and $A_{i, j}, k_{i, j}(t, t)$ and $f_{j}(t)$ are matrix functions of sizes $r_{i} \times r_{j}, r_{i} \times r_{j}$ and $r_{i} \times 1$, respectively, with $r=r_{1}+\ldots+r_{\nu}, r_{i}=r_{\nu+1-i}$ and $r_{i}=r_{\nu-i}$, $i=1, \ldots, \nu-1$, which imply $r_{1}=r_{2}=\ldots=r_{\nu}$. Hence, we use the symbol $r$ for the size of a system and the symbol $r_{1}$ instead of $r_{i}$ for $i=1, \ldots, \nu$. For the sake of simplicity, we consider the following system of Volterra 
integral equations of the first kind for $\nu=1$ :

$$
\int_{0}^{t} k_{1,1}(t, s) y_{1}(s) \mathrm{d} s=f_{1}(t),
$$

and we suppose that $k_{1,1}$ is an invertible $r \times r$ matrix-valued.

There are different notions of index for classification of IAEs. For example, Gear (1990) introduced a differential index for IAEs. The left index for (1) is another notion that was used firstly by Russian mathematicians (Bulatov, 1994; Chistyakov, 1996). For the 'degree' of ill-posedness, Lamm (2005) as well as Lamm and Scofield (2000) introduced 'v-smoothing' for Volterra integral equations of the first kind, which is equivalent with a differential index. The tractable index 1 and 2 problems are defined respectively by Brunner (2004) and Hadizadeh et al. (2011).

Piecewise polynomial collocation methods are popular methods for solving various types of operator equations, such as integral, differential and partial differential equations. From many existing papers on this subject, those which are close to our study the ones by Brunner (2004; 1978; 1977), De Hoog and Weiss (1973a; 1973b), Kauthen and Brunner (1997), as well as Weiss (1972). Piecewise (discontinuous) polynomial collocation methods for IAEs with differential index 1 of the form

$$
\begin{aligned}
y(t)+K_{11} y(t)+K_{12} z(t) & =q_{1}(t), \\
K_{21} y(t)+K_{22} z(t) & =q_{2}(t),
\end{aligned}
$$

where $K_{i, j} y(t)=\int_{0}^{t} k_{i j}(t, s) y(s) \mathrm{d} s$ for $i, j \in\{1,2\}$ with det $k_{i j} \neq 0$, were investigated by Kauthen (1997; 2001). He showed that the order of the error for these methods is $m$ and $m-1$ if the stability function

$$
\mathbb{R}(\infty)=(-1)^{m} \prod_{i=1}^{m} \frac{1-c_{i}}{c_{i}}
$$

respectively satisfies the condition $\mathbb{R}(\infty) \in[-1,1)$ and $R(\infty)=1$, where $c_{i}, \quad i=1, \ldots, m$, are the collocation parameters. The paper deals with application of the piecewise polynomial collocation method to higher index IAEs of Hessenberg form, since there are fewer investigations on these equations and their analysis is not as easy as that of index one IAEs.

The paper is organized as follows. In Section 2, we introduce a new definition based on the left index. In Section 3, we recall application of the piecewise polynomial collocation method for the system (1). In Section 4, we introduce generalized difference inequalities for supporting our analysis. In Section 5, a global convergence theorem is proved which implies the convergent properties of the given methods for the research problem given by Brunner (2004, p. 499). In
Section 6, we extend the results to the nonlinear case. Finally, in Section 7, we illustrate the obtained results by numerical experiment.

\section{Existence and uniqueness of the solution}

The existence and uniqueness theorems for the solution of IAEs depend on the definitions of the index. One of the definitions for the index of IAE, was introduced by Gear using index reduction procedure (Gear, 1990).

Definition 1. The differential index of the system (1) is $m$ ind $_{d}=m$ ), if $m$ is the minimum possible number of differentiatons of (1) required to obtain a system Volterra integral equations of the second kind.

By accepting this definition, in order to find the index of a given IAE, we must use a new proof associated with it. So there may exist different concepts of the index which are well formulated and working with them is simple. The left regularization index is one of them that was introduced by Bulatov and his collaborators (Bulatov, 2002; 1994; Chistyakov, 1996).

This definition and related powerful theorems make the investigation of existence and uniqueness results for solutions of IAEs comfortable. It is clear that for each IAE of the left index $m$ we have ind ${ }_{d}=m$, but its converse has not been investigated yet.

Definition 2. (Chistyakov, 1987) The matrix pencil $\lambda A(t)+k(t, t)$ satisfies the 'rank-degree' criterion on the interval $I$, if $\operatorname{rank} A(t)=\operatorname{deg} \operatorname{det}(\lambda A(t)+k(t, t))=$ const $>0$, for all $t \in I$.

The following conditions are necessary and sufficient for the existence of a semi-inverse matrix $A^{-}(t)$ with elements in $C^{p}\left(I, \mathbb{R}^{r \times r}\right)$ (Chistyakov, 1996):

1. the elements of $A(t)$ belong to $C^{p}\left(I, \mathbb{R}^{r \times r}\right)$,

2. $\operatorname{rank} A(t)=$ const, $\forall t \in I$.

Definition 3. Suppose that $A \in C^{\nu}\left(I, \mathbb{R}^{r \times r}\right)$ and $k \in$ $C^{\nu}\left(D, \mathbb{R}^{r \times r}\right)$. Let

$$
\begin{aligned}
A_{0} & \equiv A, \quad k_{0} \equiv k, \quad k_{i+1}=\Lambda_{i} k_{i}, \\
\Lambda_{i} y & =\frac{\mathrm{d}}{\mathrm{d} t}\left(\left(E-A_{i}(t) A_{i}^{-}(t)\right) y\right)+y, \\
A_{i+1} & \equiv A_{i}+\left(E-A_{i}(t) A_{i}^{-}(t)\right) k_{i}(t, t) .
\end{aligned}
$$

Then we say that the 'rank degree' index of $A, k$ is $\nu$ if

$$
\begin{aligned}
& \operatorname{rank} A_{i}(t)=\mathrm{const}, \quad \forall t \in I \text { for } i=0, \ldots, \nu, \\
& \operatorname{det} A_{i}=0, \text { for } i=0, \ldots, \nu-1, \operatorname{det} A_{\nu} \neq 0 .
\end{aligned}
$$

Moreover, we say that the 'rank degree' index of the system (1) is $\nu$ (ind $_{r}=\nu$ ) if, in addition to the above hypotheses, we have $f \in C^{\nu}\left(I, \mathbb{R}^{r}\right)$ and

$$
F_{i+1} \equiv \Lambda_{i} F_{i}, \quad F_{0} \equiv f,
$$

where $E$ is an identity operator. 
Lemma 1. (Bulatov, 2002) Let $\operatorname{rank} A(t)=$ const, $\forall t \in I$ and the elements of $A(t)$ are in $\mathbf{C}^{p}\left(I, \mathbb{R}^{r \times r}\right)$. Then the initial value problem

$$
\begin{aligned}
& \left(E-A A^{-}\right) x(t)^{(p)}+x(t)=0, \\
x(0)= & x^{\prime}(0)=\ldots=x^{(p-1)}(0)=0, \quad t \in I,
\end{aligned}
$$

has only a trivial solution.

Now we can state the following uniqueness and existence theorem for higher index IAEs.

Theorem 1. Suppose the following conditions are satisfied for (1):

1. ind $_{r}=\nu \geq 1$,

2. $A(t) \in \mathbf{C}^{\nu}\left(I, \mathbb{R}^{r \times r}\right), f(t) \in \mathbf{C}^{\nu}\left(I, \mathbb{R}^{r}\right)$ and $\frac{\partial^{i} k(t, s)}{\partial t^{i}} \in \mathbf{C}\left(D, \mathbb{R}^{r \times r}\right)$, for $i=1, \ldots, \nu$,

3. $A_{i}(0) y(0)=F_{i}(0)$, for $i=0, \ldots, \nu-1$ (consistency conditions).

Then the system (1) has a unique solution on I.

Proof. The proof is based on the fact that the systems

$$
\begin{gathered}
A_{i}(t) y(t)+\int_{0}^{t} k_{i}(t, s) y(s) \mathrm{d} s=F_{i}(t), \\
t \in I:=[0, T], i=0, \ldots, \nu,
\end{gathered}
$$

are equivalent (i.e., every solution of the system $i+1$ is a solution of the system $i$ and vice versa, if the consistency conditions were satisfied). To prove this assertion, let $y$ be a solution of the system $i$ and apply the operator $\Lambda_{i}$ on the system $i$. Then the system $i+1$ will be obtained. Hence $y$ is a solution of the system $i+1$. Conversely, let $y$ be a solution of system $i+1$, and define

$$
D_{i} x=A_{i}(t) x(t)+\int_{0}^{t} k_{i}(t, s) x(s) \mathrm{d} s-F_{i}(t) .
$$

Then

$$
\begin{gathered}
\frac{\mathrm{d}\left(\left(E-A_{i}(t) A_{i}^{-}(t)\right) D_{i} y\right)}{\mathrm{d} t}+D_{i} y=0, \\
D_{i} y(0)=A_{i}(0) y(0)-F(0)=0 .
\end{gathered}
$$

Because $y$ is a solution of system $i+1$, from Lemma 1 , we conclude that $D_{i} y=0$. Since the final system is a Volterra integral one of the second kind, it has a unique solution. Therefore, a unique solution satisfies all systems in (5).

We need to check whether or not the matrix $A_{\nu}$ is invertible. To this end, we use the following lemma.
Lemma 2. (Bulatov, 1994) Let the matrix pencil $\lambda A(t)+$ $k(t, t)$ satisfy the 'rank-degree' criterion on the interval $I$. Then

$$
\operatorname{det}(A(t)+V(t) k(t, t)) \neq 0, \quad \forall t \in I,
$$

and

$$
\operatorname{det}\left(A(t)+V(t)\left(A^{\prime}(t)+k(t, t)\right)\right) \neq 0, \quad \forall t \in I,
$$

where $V(t)=E-A(t) A^{-}(t)$.

To prove the existence of the solution for the system (2), it is enough to show that its index is $\nu$, and the consistency conditions hold. The proof is given in Appendix.

\section{Collocation method for IAEs}

The contents of this section is recalled after Brunner (2004). Let

$$
I_{h}:=\left\{t_{n}: 0=t_{0}<t_{1}<\cdots<t_{N}=T\right\}
$$

be a given (not necessarily uniform) partition of $I$, and set $\sigma_{n}:=\left(t_{n}, t_{n+1}\right], \bar{\sigma}_{n}:=\left[t_{n}, t_{n+1}\right]$, with $h_{n}=t_{n+1}-$ $t_{n}(n=0,1, \ldots, N-1)$ and diameter $h=\max \left\{h_{n}\right.$ : $0 \leq n \leq N\}$. Each component of the solution of (1) is approximated by elements of the piecewise polynomial space

$$
\begin{aligned}
& \mathbb{S}_{m}^{(0)}\left(I_{h}\right):=\{v \in C(I): \\
& \left.\left.v\right|_{\bar{\sigma}_{n}} \in \pi_{m}(n=0,1, \ldots, N-1)\right\},
\end{aligned}
$$

where $\pi_{m}$ denotes the space of all (real valued) polynomials of degree not exceeding $m$. A collocation solution $u_{h} \in\left(\mathbb{S}_{m}^{(0)}\left(I_{h}\right)\right)^{r}$ for (1) is defined by the equation

$$
A(t) u_{h}(t)+\int_{0}^{t} k(t, s) u_{h}(s) \mathrm{d} s=f(t),
$$

for $t \in X_{h}=\left\{t_{n, i}:=t_{n}+c_{i} h_{n}: 0=c_{0}<c_{1}<\ldots<\right.$ $\left.c_{m} \leq 1, n=0, \cdots, N-1\right\}$ and the continuity conditions

$$
u_{n-1}\left(t_{n}\right)=u_{n}\left(t_{n}\right), \quad n=1, \ldots, N-1 .
$$

The collocation parameters $c_{i}$ completely determine the set of collocation points $X_{h}$. By defining $u_{n}=$ $\left.u_{h}\right|_{\bar{\sigma}_{n}} \in\left(\pi_{m}\right)^{r}$, we have

$$
\begin{gathered}
u_{n}\left(t_{n}+s h_{n}\right)=\sum_{j=0}^{m} L_{j}(s) U_{n, j}, \quad s \in(0,1], \\
U_{n, i}:=u\left(t_{n, i}\right),
\end{gathered}
$$

where

$$
L_{j}(v):=\prod_{\substack{k=0 \\ k \neq j}}^{m} \frac{v-c_{k}}{c_{j}-c_{k}}, \quad j=0, \ldots, m,
$$


denote the Lagrange fundamental polynomials with respect to the distinct collocation parameters $c_{i}$. By partitioning the domain of integral in (7) and changing the variables, we have

$$
\begin{aligned}
f\left(t_{n, i}\right)= & A\left(t_{n, i}\right) U_{n, i}+F_{n, i} \\
& +h \int_{0}^{c_{i}} k\left(t_{n, i}, t_{n}+s h_{n}\right) u_{n}\left(t_{n}+s h_{n}\right) \mathrm{d} s
\end{aligned}
$$

where the lag terms are defined by

$$
F_{n, i}=h \sum_{l=0}^{n-1} \int_{0}^{1} k\left(t_{n, i}, t_{l}+s h_{l}\right) u_{l}\left(t_{l}+s h_{l}\right) \mathrm{d} s .
$$

Substituting from (9) in (10), for $i=1, \ldots, m$ and using the continuity conditions $(8)$, we obtain the $r m \times r m$ system

$$
\begin{aligned}
A\left(t_{n, i}\right) & U_{n, i}+h \sum_{j=1}^{m} \int_{0}^{c_{i}} k\left(t_{n, i}, t_{n}+s h_{n}\right) L_{j}(s) \mathrm{d} s U_{n, j} \\
= & -h \int_{0}^{c_{i}} k\left(t_{n, i}, t_{n}+s h_{n}\right) L_{0}(s) \mathrm{d} s U_{n-1}\left(t_{n}\right) \\
& -F_{n, i}+f\left(t_{n, i}\right)
\end{aligned}
$$

with

$$
\begin{aligned}
F_{n, i}= & h \sum_{l=0}^{n-1} \sum_{j=1}^{m}\left(\int_{0}^{1} k\left(t_{n, i}, t_{l}+s h_{l}\right) L_{j}(s) \mathrm{d} s U_{l, j}\right. \\
& \left.+\int_{0}^{1} k\left(t_{n, i}, t_{l}+s h_{l}\right) L_{0}(s) \mathrm{d} s U_{l-1}\left(t_{l}\right)\right) .
\end{aligned}
$$

By solving the system (11), the approximate solution of (1) is determined at the collocation points and $t_{n+1}$ by

$$
u_{n}\left(t_{n+1}\right)=L_{0}(1) u_{n-1}\left(t_{n}\right)+\sum_{j=1}^{m} L_{j}(1) u_{n}\left(t_{n, j}\right) .
$$

Remark 1. To apply this method, it is necessary to compute the integrals appearing in (11) and (12). To do this, we apply the following quadrature rule by using the same collocation parameters $c_{i}, i=0, \ldots, m$, such that the order of the quadrature rule is at least the same order of the method $\left(\mathcal{O}\left(h^{m+1}\right)\right)$,

$$
\begin{gathered}
\int_{0}^{c_{i}} k\left(t_{n, i}, t_{n}+s h_{n}\right) L_{j}(s) \mathrm{d} s \simeq a_{i, j} k\left(t_{n, i}, t_{n}+c_{j} h_{n}\right), \\
\int_{0}^{1} k\left(t_{n, i}, t_{l}+s h_{l}\right) L_{j}(s) \mathrm{d} s \simeq b_{j} k\left(t_{n, i}, t_{l}+c_{j} h_{l}\right),
\end{gathered}
$$

with $a_{i, j}=\int_{0}^{c_{i}} L_{j}(t) \mathrm{d} t$ and $b_{j}=\int_{0}^{1} L_{j}(t) \mathrm{d} t$. Using this quadrature rule considerably simplifies our computations. When all the integrals are computed by the quadrature rule, the method is called fully discretised.
Remark 2. Choosing $c_{m}=1$, we have $t_{n+1}=t_{n, m}$ and $u\left(t_{n+1}\right)=u\left(t_{n, m}\right)$. Thus we obtain $u_{n+1}=$ $U_{n, m}$ without reusing (9). This also makes the analysis of existence and uniqueness of the approximate solution simple, which will be discussed in the next section.

Some existence and uniqueness conditions for the solution of continuous collocation methods can be found in the work of Brunner (2004).

\section{Difference inequalities}

Firstly, we recall the following lemmas. Note that we write $v=O\left(h^{m}\right)$ whenever $\|v\|=O\left(h^{m}\right)$.

Lemma 3. (Gronwall's inequality (Brunner, 2004)) Assume that $\left\{k_{j}\right\},(j \geq 0)$ is a given non-negative sequence, and the sequence $\left\{\epsilon_{n}\right\}$ satisfies $\epsilon_{0} \leq \rho_{0}$ and

$$
\epsilon_{n} \leq \rho_{0}+\sum_{j=0}^{n-1} q_{j}+\sum_{j=0}^{n-1} k_{j} \epsilon_{j}
$$

with $\rho_{0} \geq 0, q_{j} \geq 0,(j \geq 0)$. Then

$$
\epsilon_{n} \leq\left(\rho_{0}+\sum_{j=0}^{n-1} q_{j}\right) \exp \left(\sum_{j=0}^{n-1} k_{j}\right)
$$

Lemma 4. Let $B_{j}, j \geq 0$, be a uniformly bounded sequence of $v \times v$ matrices, $M=\operatorname{diag}\left(\lambda_{1}, \ldots, \lambda_{v}\right)$ and $\lambda=\max _{i}\left|\lambda_{i}\right|$. Let also $\left\{E_{n}\right\}$ be a set of vectors with

$$
E_{0}=\mathcal{O}\left(h^{u_{1}}\right)
$$

and

$$
E_{n} \leq M E_{n-1}+h \sum_{l=0}^{n-1} B_{l} E_{l}+\mathcal{O}\left(h^{u_{2}}\right), \quad n=1, \ldots, N \text {. }
$$

Then

$$
\lim _{\substack{N \rightarrow \infty \\ N h=\text { const }}}\left\|E_{N}\right\|=\infty
$$

for $\lambda>1$, and

$$
\lim _{\substack{N \rightarrow \infty \\ N h=\text { const }}}\left\|E_{N}\right\| \leq \mathcal{O}\left(h^{\min \left\{u_{1}, u_{2}\right\}}\right)
$$

for $\lambda_{i} \in[-1,1), i=1, \ldots, v$.

Proof. The proof can be derived easily by using Gronwall's inequality.

\section{Convergence analysis}

Before stating the convergence properties of continuous collocation methods, we investigate the convergence properties of perturbed continuous collocation methods 
for a System of First kind Volterra Integral Equations (SFVIE). The proof of Theorem 2 in this section is similar to the standard technique introduced by De Hoog and Weiss (1973a; 1973b), Brunner (1978; 1977), as well as Kauthen and Brunner (1997). An excellent book for this technique is the one by Brunner (2004). However, for the convenience of the readers and self-dependency of the paper, we give the proofs of all theorems in detail.

Consider the SFVIE

$$
\int_{0}^{t} k(t, s) y(s) \mathrm{d} s=f(t)
$$

where $k(t, t)$ is an invertible $r \times r$ matrix for all $t \in I$. We analyze the convergence properties of the perturbed continuous spline collocation method. For solving the SFVIE, we perturb the system (11) as

$$
\begin{aligned}
h \sum_{j=1}^{m} & \int_{0}^{c_{i}} k\left(t_{n, i}, t_{n}+s h_{n}\right) L_{j}(s) \mathrm{d} s U_{n, j} \\
= & -h \int_{0}^{c_{i}} k\left(t_{n, i}, t_{n}+s h_{n}\right) L_{0}(s) \mathrm{d} s U_{n-1}\left(t_{n}\right) \\
& -F_{n, i}+f\left(t_{n, i}\right)+\delta(h, n, i),
\end{aligned}
$$

where

$$
\begin{aligned}
F_{n, i}= & h \sum_{l=0}^{n-1} \sum_{j=1}^{m}\left(\int_{0}^{1} k\left(t_{n, i}, t_{l}+s h_{l}\right) L_{j}(s) \mathrm{d} s U_{l, j}\right. \\
& \left.+\int_{0}^{1} k\left(t_{n, i}, t_{l}+s h_{l}\right) L_{0}(s) \mathrm{d} s U_{l-1}\left(t_{l}\right)\right)
\end{aligned}
$$

with

$$
F_{0, i}=0 .
$$

Here, the perturbed term $\delta(h, n, i)$ only depends on $h$ and $t_{n, i}$, and it is of order $\mathcal{O}\left(h^{m_{1}}\right)$.

Theorem 2. Let

$$
f(t) \in C^{m+2}\left(I, \mathbb{R}^{r}\right), \quad \frac{\partial^{i} k(t, s)}{\partial t^{i}} \in \mathbf{C}\left(D, \mathbb{R}^{r \times r}\right)
$$

for $i=1, \ldots, m+1$, satisfy the system (15). Then the approximate solution $u_{h}$ of the perturbed continuous collocation method with distinct collocation parameters $c_{1}, \ldots, c_{m} \in(0,1]$ and $c_{m} \leq 1$ (if exists) converges to the solution $y$ for any $m \geq 2$ if and only if

$$
\varrho=\max \left\{\left|\lambda_{1}\right|,\left|\lambda_{2}\right|\right\} \leq 1,
$$

where $\lambda_{1}$ and $\lambda_{2}$ are the eigenvalues of the matrix $\widetilde{A}^{-1} B$ with

$$
\widetilde{A}=\left(\begin{array}{cccc}
1 & 0 & \ldots & 0 \\
a_{10} & a_{11} & \ldots & a_{11} \\
\vdots & \vdots & \ddots & \vdots \\
a_{m 0} & a_{m 1} & \ldots & a_{m m}
\end{array}\right)
$$

and

$$
B=\left(\begin{array}{cccc}
L_{0}(1) & L_{1}(1) & \ldots & L_{m}(1) \\
a_{m 0}-b_{0} & a_{m 1}-b_{1} & \ldots & a_{m m}-b_{m} \\
\vdots & \vdots & \ddots & \vdots \\
a_{m 0}-b_{0} & a_{m 1}-b_{1} & \ldots & a_{m m}-b_{m}
\end{array}\right),
$$

and the collocation error satisfies

$$
\begin{aligned}
& \left\|y-u_{h}\right\| \\
& \leq \begin{cases}\mathcal{O}\left(h^{\min \left\{m_{1}-1, m+1\right\}}\right) & \text { if } \lambda_{1}, \lambda_{2} \in[-1,1), \\
\mathcal{O}\left(h^{\min \left\{m_{1}-2, m\right\}}\right) & \text { if } \lambda_{1}=1 \text { or } \lambda_{2}=1 .\end{cases}
\end{aligned}
$$

Remark 3. The eigenvalues $\lambda_{1}$ and $\lambda_{2}$ can be computed by

$$
\begin{aligned}
& \lambda_{1}=\frac{1}{2}\left(\operatorname{tr}\left(\widetilde{A}^{-1} B\right)+\sqrt{\left(\operatorname{tr}\left(\widetilde{A}^{-1} B\right)\right)^{2}-4\left(L_{0}(1)\right)^{2}}\right), \\
& \lambda_{2}=\frac{1}{2}\left(\operatorname{tr}\left(\widetilde{A}^{-1} B\right)-\sqrt{\left(\operatorname{tr}\left(\widetilde{A}^{-1} B\right)\right)^{2}-4\left(L_{0}(1)\right)^{2}}\right),
\end{aligned}
$$

where

$$
\operatorname{tr}\left(\widetilde{A}^{-1} B\right)=L_{0}(1)\left(2+\sum_{i=1}^{m} \frac{1}{c_{i}}+\sum_{i=1}^{m} \frac{1}{1-c_{i}}\right),
$$

for $c_{m}<1$ and

$$
\begin{gathered}
\lambda_{1}=0, \\
\lambda_{2}=(-1)^{m} \prod_{i=1}^{m-1} \frac{1-c_{i}}{c_{i}},
\end{gathered}
$$

for $c_{m}=1$ (see Kauthen and Brunner, 1997).

Proof. Solving (16) and (17) is equivalent to finding $u_{h}(s) \in\left(\mathbb{S}_{m}^{(0)}\left(I_{h}\right)\right)^{r}$ such that

$$
\int_{0}^{t_{n i}} k\left(t_{n i}, s\right) u_{h}(s) \mathrm{d} s=f\left(t_{n i}\right)+\delta(h, n, i) .
$$

Subtracting this equation from (15) with $t=t_{n, i}$, we obtain

$$
\int_{0}^{t_{n i}} k\left(t_{n i}, s\right) e(s) \mathrm{d} s=\delta(h, n, i),
$$

where $e(s)=y(s)-u(s)$. Introducing $e_{n}=\left.e\right|_{\bar{\sigma}_{n}} \in$ $C^{m+1}\left[t_{n}, t_{n+1}\right]$, we have

$e_{n}\left(t_{n}+v h\right)=e_{n-1}\left(t_{n}\right) L_{0}(v)+\sum_{j=1}^{m} L_{j}(v) e_{n}\left(t_{n, j}\right)+r_{n}(v)$,

$v \in[0,1]$, where the interpolation error is determined by

$$
\begin{aligned}
& r_{n}(v) \\
= & h^{m+1} \frac{y^{(m+1)}\left(\xi_{n}(v)\right)}{(m+1) !} v \prod_{i=1}^{m}\left(v-c_{i}\right) \quad \xi_{n}(v) \in\left(t_{n}, t_{n+1}\right) .
\end{aligned}
$$


(Notice that we have $f(t) \in C^{m+2}\left(I, \mathbb{R}^{r}\right), \frac{\partial^{i} k(t, s)}{\partial t^{i}} \in$ $\mathbf{C}\left(D, \mathbb{R}^{r \times r}\right)$, for $i=1, \ldots, m+1$, by assumption.)

From (24), we have

$$
\begin{aligned}
& \int_{0}^{c_{i}} k\left(t_{n i}, t_{n}+s h\right) e\left(t_{n}+s h\right) \mathrm{d} s \\
& =-\sum_{l=0}^{n-1} \int_{0}^{1} k\left(t_{n i}, t_{l}+s h\right) e\left(t_{l}+s h\right) \mathrm{d} s \\
& \quad+\frac{\delta(h, n, i)}{h} .
\end{aligned}
$$

Rewriting (27) with $n$ replaced by $n-1$ and $j=m$ and subtracting it from (27), we obtain

$$
\begin{aligned}
& \int_{0}^{c_{i}} k\left(t_{n i}, t_{n}+s h\right) e_{n}\left(t_{n}+s h\right) \mathrm{d} s \\
& =\int_{0}^{c_{m}} k\left(t_{n-1, m}, t_{n-1}+s h\right) e_{n-1}\left(t_{n-1}+s h\right) \mathrm{d} s \\
& \quad-\int_{0}^{1} k\left(t_{n i}, t_{n-1}+s h\right) e_{n-1}\left(t_{n-1}+s h\right) \mathrm{d} s \\
& \quad+\sum_{l=0}^{n-2} \int_{0}^{1}\left(k\left(t_{n-1, m}, t_{l}+s h\right)\right. \\
& \left.\quad-k\left(t_{n i}, t_{l}+s h\right)\right) e_{l}\left(t_{l}+s h\right) \mathrm{d} s \\
& +\frac{\delta(h, n, i)-\delta(h, n-1, m)}{h} .
\end{aligned}
$$

Using

$$
\begin{gathered}
\int_{0}^{c_{i}} k\left(t_{n i}, t_{n}+s h\right) L_{j}(s) \mathrm{d} s \\
=k\left(t_{n}, t_{n}\right) a_{i j}+\mathcal{O}(h), \\
\int_{0}^{c_{m}} k\left(t_{n-1, m}, t_{n-1}+s h\right) L_{j}(s) \mathrm{d} s \\
=k\left(t_{n}, t_{n}\right) a_{m j}+\mathcal{O}(h), \\
\int_{0}^{1} k\left(t_{n i}, t_{n-1}+s h\right) L_{j}(s) \mathrm{d} s \\
=k\left(t_{n}, t_{n}\right) b_{j}+\mathcal{O}(h)
\end{gathered}
$$

and

$$
\begin{aligned}
& k\left(t_{n-1, m}, t_{l}+s h\right)-k\left(t_{n i}, t_{l}+s h\right) \\
& \quad=h\left(1+c_{i}-c_{m}\right) k_{t}\left(\xi_{n}, t_{l}\right)+\mathcal{O}(h)
\end{aligned}
$$

in 28, we obtain

$$
\begin{aligned}
& \left(k\left(t_{n}, t_{n}\right)+\mathcal{O}(h)\right)\left(\sum_{j=0}^{m} a_{i j} e_{n}\left(t_{n j}\right)+\mathcal{O}\left(h^{m+1}\right)\right) \\
& =\left(k\left(t_{n}, t_{n}\right)+\mathcal{O}(h)\right)
\end{aligned}
$$

$$
\begin{aligned}
& \times\left(\sum_{j=0}^{m} a_{m j} e_{n-1}\left(t_{n-1, j}\right)+\mathcal{O}\left(h^{m+1}\right)\right) \\
& -\left(k\left(t_{n}, t_{n}\right)+\mathcal{O}(h)\right) \\
& \times\left(\sum_{j=0}^{m} b_{j} e_{n-1}\left(t_{n-1, j}\right)+\mathcal{O}\left(h^{m+1}\right)\right) \\
& +\left(h\left(1+c_{i}-c_{m}\right) k_{t}\left(\xi_{n}, t_{l}\right)+\mathcal{O}(h)\right) \\
& \times \sum_{l=0}^{n-2}\left(\sum_{j=0}^{m} b_{j} e_{l}\left(t_{l, j}\right)+\mathcal{O}\left(h^{m+1}\right)\right) \\
& +\frac{\delta(h, n, i)-\delta(h, n-1, m)}{h} .
\end{aligned}
$$

Since $k(t, t)$ is invertible and continuous with respect to $t, k\left(t_{n}, t_{n}\right)+\mathcal{O}(h)$ has a continuous inverse, say $W$, for sufficiently small $h$. Hence, Eqn. (29) can be written as

$$
\begin{aligned}
& \sum_{j=0}^{m} a_{i j} e_{n}\left(t_{n j}\right) \\
& =\sum_{j=0}^{m} a_{m j} e_{n-1}\left(t_{n-1, j}\right) \\
& \quad-\sum_{j=0}^{m} b_{j} e_{n-1}\left(t_{n-1, j}\right)+h W U \sum_{l=0}^{n-2} \sum_{j=0}^{m} b_{j} e_{l}\left(t_{l, j}\right) \\
& \quad+\mathcal{O}\left(h^{m_{1}-1}\right)+\mathcal{O}\left(h^{m+1}\right),
\end{aligned}
$$

where $h U:=h\left(1+c_{i}-c_{m}\right) k_{t}\left(\xi_{n}, t_{l}\right)+\mathcal{O}(h)$ and so $U$ is bounded with respect to its variables. Using 25) and the continuity conditions $(8)$, we have

$$
\begin{aligned}
e_{n}\left(t_{n}\right)= & e_{n-1}\left(t_{n-1}+h\right) \\
= & e_{n-1}\left(t_{n-1}\right) L_{0}(1) \\
& +\sum_{j=1}^{m} L_{j}(1) e_{n-1}\left(t_{n-1, j}\right)+r_{n-1}(1) .
\end{aligned}
$$

Thus from Eqns. (31) and (30), we can write

$$
\begin{gathered}
\left(\begin{array}{cccc}
\mathbf{I} & O & \ldots & O \\
a_{10} \mathbf{I} & a_{11} \mathbf{I} & \ldots & a_{1 m} \mathbf{I} \\
\vdots & \vdots & \ddots & \vdots \\
a_{m 0} \mathbf{I} & a_{m 1} \mathbf{I} & \ldots & a_{m m} \mathbf{I}
\end{array}\right)\left(\begin{array}{c}
e_{n}\left(t_{n}\right) \\
e_{n}\left(t_{n, 1}\right) \\
\vdots \\
e_{n}\left(t_{n, m}\right)
\end{array}\right) \\
=\left(\begin{array}{ccc}
L_{0}(1) \mathbf{I} & \ldots & L_{m}(1) \mathbf{I} \\
\left(a_{m 0}-b_{0}\right) \mathbf{I} & \ldots & \left(a_{m m}-b_{m}\right) \mathbf{I} \\
\vdots & & \ddots \\
\left(a_{m 0}-b_{0}\right) \mathbf{I} & \ldots & \left(a_{m m}-b_{m}\right) \mathbf{I}
\end{array}\right)
\end{gathered}
$$




$$
\begin{aligned}
& \times\left(\begin{array}{c}
e_{n-1}\left(t_{n-1}\right) \\
e_{n-1}\left(t_{n-1,1}\right) \\
\vdots \\
e_{n-1}\left(t_{n-1, m}\right)
\end{array}\right) \\
+ & h \sum_{l=0}^{n-2} B_{l} E_{l}+\mathcal{O}\left(h^{m_{1}-1}\right)+\mathcal{O}\left(h^{m+1}\right),
\end{aligned}
$$

where $O$ and $\mathbf{I}$ are zero and identity $r \times r$ matrices, respectively, $E_{l}=\left[e_{l}\left(t_{l, 0}\right), \ldots, e_{l}\left(t_{l, m}\right)\right]$ and $B_{l}$ are appropriate matrices. By using the Kronecker product, we summarize Eqn. (32) in the form

$$
(\widetilde{A} \otimes \mathbf{I}) E_{n}=(B \otimes \mathbf{I}) E_{n-1}+h \sum_{l=0}^{n-2} B_{l} E_{l}+\mathcal{O}\left(h^{u}\right),
$$

$u=\min \left\{m_{1}-1, m+1\right\}$. Since $\widetilde{A}$ and $\mathbf{I}$ are invertible matrices, we have

$E_{n}=\left(\left(\widetilde{A}^{-1} B\right) \otimes \mathbf{I}\right) E_{n-1}+h \sum_{l=0}^{n-2}\left(\widetilde{A}^{-1} \otimes \mathbf{I} B_{l}\right) E_{l}+\mathcal{O}\left(h^{u}\right)$.

After Kauthen and Brunner (1997, Lemma 2), there exists an invertible matrix $P$ such that $D:=$ $P\left(\widetilde{A}^{-1} B\right) P^{-1}=\operatorname{diag}\left(\lambda_{1}, \lambda_{2}, 0, \ldots, 0\right)$. Thus, we can use $(P \otimes \mathbf{I})\left(\left(\widetilde{A}^{-1} B\right) \otimes \mathbf{I}\right)(P \otimes \mathbf{I})^{-1}(P \otimes \mathbf{I})=D \otimes \mathbf{I}$ to write (34) as

$$
\begin{aligned}
(P \otimes \mathbf{I}) E_{n} & (D \otimes \mathbf{I})(P \otimes \mathbf{I}) E_{n-1} \\
& +h \sum_{l=0}^{n-2}\left(\widetilde{A}^{-1} \otimes \mathbf{I}\right) B_{l}(P \otimes \mathbf{I})^{-1}(P \otimes \mathbf{I}) E_{l} \\
& +\mathcal{O}\left(h^{u}\right),
\end{aligned}
$$

where $\lambda_{1}$ and $\lambda_{2}$, are obtained by (18)- 20). It is obvious that $E_{0}=\mathcal{O}\left(h^{\min \left\{m_{1}, m+1\right\}}\right)$. Thus we can use Lemma4 to prove

$$
\lim _{\substack{N \rightarrow \infty \\ N h=\text { const }}}\left\|(P \otimes \mathbf{I}) E_{N}\right\|=\leq \mathcal{O}\left(h^{\min \left\{m_{1}-1, m+1\right\}}\right),
$$

and by using (25) the proof is completed for the case $\lambda_{1}, \lambda_{2} \in[-1,1)$.

For the case $\lambda_{1}=1$ or $\lambda_{2}=1$, we apply the given ideas of Kauthen and Brunner (1997) to use the interpolation formula for $e(t)$ at a smaller number of collocation points, but this application in the perturbation case is not as simple as the ideas of Kauthen and Brunner (1997) and we need the following remarks.

Remark 4. Let $a_{i j}=\int_{0}^{c_{i}} \bar{L}_{j}(s) \mathrm{d} s$ and $b_{j}=\int_{0}^{1} \bar{L}_{j}(s) \mathrm{d} s$, where $\bar{L}_{j}(v), j=1, \ldots, l$, are the Lagrange polynomials of degree $l$ defined with respect to the points $0<d_{1}<$ $\ldots<d_{l}<1$. Then $M=A^{-1} B$ has only the eigenvalue

$$
\bar{R}(\infty)=(-1)^{l} \prod_{i=1}^{l} \frac{1-d_{i}}{d_{i}}
$$

where $A=\left(a_{i j}\right)$ and $B=\left(B_{i j}\right)$ with $B_{i j}=a_{m j}-b_{j}$. Moreover,

$e\left(t_{n}+v h\right)=\sum_{j=1}^{l} \bar{L}_{j}(v) e_{n}\left(\bar{t}_{n, j}\right)+h^{m} \frac{e_{n}^{(l)}\left(\bar{t}_{n}^{+}\right)}{l !} \prod_{i=1}^{l}\left(\tau-c_{i}\right)$,

$\tau \in[0,1]$, where $\bar{t}_{n, j}=t_{n}+d_{j} h$, (see, e.g., Kauthen and Brunner, 1997).

Remark 5. For the case $\lambda_{1}=1$ or $\lambda_{2}=1$, we know the matrix $M$ from Remark 4 with respect to the points $c_{1}, \ldots, c_{m}$ has the only eigenvalue

$$
\bar{R}(\infty)=(-1)^{m} \prod_{i=1}^{m} \frac{1-c_{i}}{c_{i}}<1
$$

(see Kauthen and Brunner, 1997). Hence it can be easily proved that, for each $l \leq m$, we can choose $l$ points $d_{1}, \ldots, d_{l}$ through $c_{1}, \ldots, c_{m}$ such that

$$
\bar{R}(\infty)=(-1)^{l} \prod_{i=1}^{l} \frac{1-d_{i}}{d_{i}}<1 .
$$

Let $l_{1}=\min \left\{m_{1}-1, m+1\right\}$. Then we use the above remarks with $l=l_{1}-1$ and the collocation points $d_{1}, \ldots, d_{l}$ to get

$$
e_{n}\left(t_{n}+v h\right)=\sum_{j=1}^{l} \bar{L}_{j}(v) e_{n}\left(t_{n, j}\right)+O\left(h^{l}\right), \quad v \in[0,1] .
$$

By substituting (37) into (28) and following the lines of the proof after Eqn. (28), we obtain

$$
E_{n}=(M \otimes \mathbf{I}) E_{n-1}+h \sum_{l=0}^{n-1} \overline{B_{n l}} E_{l}+O\left(h^{l}\right) .
$$

Since $M$ is diagonalizable, there exists $P$ such that $D=$ $P M P^{-1}=\operatorname{diag}(\bar{R}(\infty), 0, \ldots, 0)$ with $|\bar{R}(\infty)|<1$. Now applying Lemma 4 to the equation

$$
\begin{aligned}
(P \otimes \mathbf{I}) E_{n}= & (M \otimes \mathbf{I})(P \otimes \mathbf{I}) E_{n-1} \\
& +h \sum_{l=0}^{n-1}(P \otimes \mathbf{I}) \overline{B_{n l}} E_{l}+O\left(h^{l}\right)
\end{aligned}
$$

completes the proof.

Remark 6. The trivial conclusion of Theorem 2 is for the case $\delta(h, n, i)=0$, (e.g., $m_{1}=\infty$ ). This case is equivalent to using the continuous collocation method for the system of first kind Volterra integral equations, where one can use this theorem to prove

$$
\|e\| \leq \begin{cases}\mathcal{O}\left(h^{m+1}\right) & \text { if } \lambda_{1}, \lambda_{2} \in[-1,1), \\ \mathcal{O}\left(h^{m}\right) & \text { if } \lambda_{1}=1 \text { or } \lambda_{2}=1,\end{cases}
$$

which is the same as the result of Kauthen and Brunner (1997) for the Volterra integral equation of the first kind. 
This perturbed analysis on the numerical solution of the Volterra integral equations of the first kind makes it easy for the Hessenberg form (we claim that without this perturbation analysis, the proof of the next theorem will be more complicated).

Theorem 3. Let $f(t) \in C^{m+2}\left(I, \mathbb{R}^{r}\right), \frac{\partial^{i} k(t, s)}{\partial t^{i}} \in$ $\mathbf{C}\left(D, \mathbb{R}^{r \times r}\right) \quad$ for $i=1, \ldots, m+1$ and $A(t) \in$ $\mathbf{C}^{m+1}\left(I, \mathbb{R}^{r \times r}\right)$ be satisfied for the system (1) and suppose that the system has Hessenberg form of index $\nu$ (see Eqn. (2)). Also suppose that the consistency conditions of Theorem $\square$ hold for this system. Then the approximate solution $u_{h}$ of the continuous collocation method with distinct collocation parameters $c_{1}, \ldots, c_{m} \in(0,1]$ (if it exists) converges to the solution $y$ if and only if

$$
\varrho=\max \left\{\left|\lambda_{1}\right|,\left|\lambda_{2}\right|\right\} \leq 1,
$$

where $\lambda_{1}$ and $\lambda_{2}$ are given by (18)-(22), and the collocation error satisfies

$$
\begin{aligned}
& \left\|y_{1}-u_{1}\right\| \leq \begin{cases}\mathcal{O}\left(h^{m+1}\right) & \text { if } \lambda_{1}, \lambda_{2} \in[-1,1), \\
\mathcal{O}\left(h^{m}\right) & \text { if } \lambda_{1}=1 \text { or } \lambda_{2}=1,\end{cases} \\
& \left\|y_{2}-u_{2}\right\| \leq \begin{cases}\mathcal{O}\left(h^{m}\right) & \text { if } \lambda_{1}, \lambda_{2} \in[-1,1), \\
\mathcal{O}\left(h^{m-2}\right) & \text { if } \lambda_{1}=1 \text { or } \lambda_{2}=1\end{cases}
\end{aligned}
$$

$\left\|y_{\nu}-u_{\nu}\right\| \leq \begin{cases}\mathcal{O}\left(h^{m+2-\nu}\right) & \text { if } \lambda_{1}, \lambda_{2} \in[-1,1) \\ \mathcal{O}\left(h^{m+2-2 \nu}\right) & \text { if } \lambda_{1}=1 \text { or } \lambda_{2}=1\end{cases}$

and hence

$$
\left\|y-u_{h}\right\| \leq \begin{cases}\mathcal{O}\left(h^{m+2-\nu}\right) & \text { if } \lambda_{1}, \lambda_{2} \in[-1,1) \\ \mathcal{O}\left(h^{m+2-2 \nu}\right) & \text { if } \lambda_{1}=1 \text { or } \lambda_{2}=1\end{cases}
$$

where $u_{h}=\left[u_{1}, \ldots, u_{\nu}\right]^{T}$.

Proof. The proof is derived by induction on $\nu$. For the case $\nu=1$, it is trivial (Remark [). Suppose that it is true for $\nu=n$. For $\nu=n+1$, the problem takes the form

$$
\begin{aligned}
& {\left[\begin{array}{cccc}
A_{1,1}(t) & \ldots & A_{1, n}(t) & 0 \\
\vdots & \ddots & \vdots & \vdots \\
A_{n, 1}(t) & \ldots & 0 & 0 \\
0 & \ldots & 0 & 0
\end{array}\right]\left[\begin{array}{c}
y_{1}(t) \\
y_{2}(t) \\
\vdots \\
y_{n+1}(t)
\end{array}\right]} \\
& +\int_{0}^{t}\left[\begin{array}{cccc}
k_{1,1}(t, s) & k_{1,2}(t, s) & \ldots & k_{1, n+1}(t, s) \\
\vdots & \vdots & \ddots & \vdots \\
k_{n, 1}(t, s) & k_{k, 2}(t, s) & \ldots & 0 \\
k_{n+1,1}(t, s) & 0 & 0
\end{array}\right] \\
& \times\left[\begin{array}{c}
0 \\
y_{1}(t) \\
y_{2}(t) \\
\vdots \\
y_{n+1}(t)
\end{array}\right] \mathrm{d} s=\left[\begin{array}{c}
f_{1}(t) \\
f_{2}(t) \\
\vdots \\
f_{n+1}(t)
\end{array}\right] .
\end{aligned}
$$

The last $n$ equations of this system form an IAE of index $n$. By the hypothesis of induction, we have

$$
\begin{gathered}
\left\|e_{1}\right\| \leq \begin{cases}\mathcal{O}\left(h^{m+1}\right) & \text { if } \lambda_{1}, \lambda_{2} \in[-1,1), \\
\mathcal{O}\left(h^{m}\right) & \text { if } \lambda_{1}=1 \text { or } \lambda_{2}=1,\end{cases} \\
\left\|e_{2}\right\| \leq \begin{cases}\mathcal{O}\left(h^{m}\right) & \text { if } \lambda_{1}, \lambda_{2} \in[-1,1), \\
\mathcal{O}\left(h^{m-2}\right) & \text { if } \lambda_{1}=1 \text { or } \lambda_{2}=1,\end{cases} \\
\quad \vdots \\
\left\|e_{n}\right\| \leq \begin{cases}\mathcal{O}\left(h^{m+2-n}\right) & \text { if } \lambda_{1}, \lambda_{2} \in[-1,1), \\
\mathcal{O}\left(h^{m+2-2 n}\right) & \text { if } \lambda_{1}=1 \text { or } \lambda_{2}=1,\end{cases} \\
\qquad \begin{array}{ll}
\text { here } \left.e_{i}=y_{i}-u_{i} \text { for } i=1, \ldots, n\right), \text { and hence }
\end{array} \\
\mid A_{1,1}(t) e_{1}(t)+\ldots+A_{1, n}(t) e_{n}(t) \\
+\int_{0}^{t} k_{1,1}(t, s) e_{1}(s) \text { d } s \ldots+\int_{0}^{t} k_{1, n}(t, s) e_{n}(s) \mathrm{d} s \mid \\
\leq \begin{cases}\mathcal{O}\left(h^{m+2-n}\right) & \text { if } \lambda_{1}, \lambda_{2} \in[-1,1), \\
\mathcal{O}\left(h^{m+2-2 n}\right) & \text { if } \lambda_{1}=1 \text { or } \lambda_{2}=1 .\end{cases}
\end{gathered}
$$

The first equation of the system (38) is a Volterra integral equation of the first kind, i.e.,

$$
\begin{aligned}
\int_{0}^{t} k_{1, n+1}(t, s) y_{n+1}(s) \mathrm{d} s \\
=f_{1}(t)-A_{1,1}(t) y_{1}(t)+\cdots-A_{1, n}(t) y_{n}(t) \\
\quad-\int_{0}^{t} k_{1,1}(t, s) y_{1}(s) \mathrm{d} s-\cdots \\
\quad-\int_{0}^{t} k_{1, n}(t, s) y_{n}(s) \mathrm{d} s .
\end{aligned}
$$

From [39, the corresponding continuous collocation method for Eqn. (40) is equivalent to the perturbed continuous collocation method with the perturbation term

$$
\delta(h, n, i)= \begin{cases}\mathcal{O}\left(h^{m+2-n}\right) & \text { if } \lambda_{1}, \lambda_{2} \in[-1,1) \\ \mathcal{O}\left(h^{m+2-2 n}\right) & \text { if } \lambda_{1}=1 \text { or } \lambda_{2}=1\end{cases}
$$

Then Theorem 2 yields

$$
\begin{aligned}
\| y_{n+1} & -u_{n+1} \| \\
& \leq \begin{cases}\mathcal{O}\left(h^{m+1-n}\right) & \text { if } \lambda_{1}, \lambda_{2} \in[-1,1), \\
\mathcal{O}\left(h^{m-2 n}\right) & \text { if } \lambda_{1}=1 \text { or } \lambda_{2}=1,\end{cases}
\end{aligned}
$$

which completes the proof.

The fully discretised perturbed continuous collocation solution $\widehat{u}_{n}\left(t_{n}+s h_{n}\right)=\sum_{j=0}^{m} L_{j}(s) \widehat{U}_{n, j}$, for the Fully Discretised Continuous Collocation Method 
(FDCCM) is obtained by solving the system

$$
\begin{aligned}
h \sum_{j=1}^{m} a_{i j} k\left(t_{n, i}, t_{n, j}\right) \widehat{U}_{n, j} \\
=-h a_{i j} k\left(t_{n, i}, t_{n, j}\right) \widehat{U}_{n-1}\left(t_{n}\right)+f\left(t_{n, i}\right) \\
\quad-h \sum_{l=0}^{n-1}\left(\sum_{j=1}^{m} b_{j} k\left(t_{n, i}, t_{n, j}\right) \widehat{U}_{l, j}\right. \\
\left.\quad+b_{0} k\left(t_{n, i}, t_{n, j}\right) \widehat{U}_{l-1}\left(t_{l}\right)\right)+\bar{\delta}(h, n, i) .
\end{aligned}
$$

The FDCCM has also the same order of convergence which we obtained for the continuous collocation method. To prove this, we need a new version of Theorem 2

Theorem 4. Let $f(t) \in C^{m+2}\left(I, \mathbb{R}^{r}\right), \frac{\partial^{i} k(t, s)}{\partial t^{i}} \in$ $\mathbf{C}\left(D, \mathbb{R}^{r \times r}\right)$ for $i=1, \ldots, m+1$ be satisfied for the system (15). Then the approximate solution $u_{h}$ of the fully discretised perturbed continuous collocation method with distinct collocation parameters $c_{1}, \ldots, c_{m} \in(0,1]$ and $c_{m} \leq 1$ (if it exists) converges to the solution y for all $m \geq 2$ if and only if

$$
\varrho=\max \left\{\left|\lambda_{1}\right|,\left|\lambda_{2}\right|\right\} \leq 1,
$$

where $\lambda_{1}$ and $\lambda_{2}$ are given by (18)-(22) and the collocation error satisfies

$$
\begin{aligned}
& \left\|y-u_{h}\right\| \\
& \leq \begin{cases}\mathcal{O}\left(h^{\min \left\{m_{1}-1, m+1\right\}}\right) & \text { if } \lambda_{1}, \lambda_{2} \in[-1,1), \\
\mathcal{O}\left(h^{\min \left\{m_{1}-2, m\right\}}\right) & \text { if } \lambda_{1}=1 \text { or } \lambda_{2}=1 .\end{cases}
\end{aligned}
$$

Proof. We can proceed similarly to the proof of Theorem 2 to show that $\left\|\widehat{u}_{h}-u_{h}\right\|$ has the same order of $\left\|y-u_{h}\right\|$. Then using

$$
\left\|y-\widehat{u}_{h}\right\| \leq\left\|\widehat{u}_{h}-u_{h}\right\|+\left\|y-u_{h}\right\|
$$

completes the proof.

Analysis of index 2 IAEs of the form (2) was introduced as a research problem by Brunner (2004), (p. 499), so by using the previous theorems we have following corollary for this problem.

Corollary 1. Consider the system of IAEs (2) of size $r=$ $r_{1}+r_{2}$ and suppose following conditions are satisfied:

1. the functions $f_{i}(t) \in \mathbf{C}^{m+1}\left(I, \mathbb{R}^{r}\right)$ and $A_{i, j}(t) \in$ $\mathbf{C}^{m+1}\left(I, \mathbb{R}^{r_{i} \times r_{j}}\right)$, for $i, j=1,2$.

2. $\partial^{l} k_{i, j}(t, s) / \partial t^{l} \in \mathbf{C}^{m+1}\left(D, \mathbb{R}^{r_{i} \times r_{j}}\right)$, for $l=$ $1, \ldots, m+1$ and $i, j=1,2$.

3. $f_{2}(0)=0$ and $f_{1}(0)=f_{2}^{\prime}(0) / k_{21}(0,0)$ (the consistency conditions).
Then the following statements hold on $I$ :

1. The system (2) has a unique solution.

2. The approximate solution $u_{h}$ of the collocation method with distinct collocation parameters $c_{1}, \ldots, c_{m} \in(0,1]$ (if exists) satisfies

$$
\left\|y-u_{h}\right\| \leq \begin{cases}\mathcal{O}\left(h^{m}\right) & \text { if } \lambda_{1}, \lambda_{2} \in[-1,1) \\ \mathcal{O}\left(h^{m-2}\right) & \text { if } \lambda_{1}=1 \text { or } \lambda_{2}=1 .\end{cases}
$$

\section{Nonlinear case}

Suppose that the equation

$$
\begin{aligned}
A(t) y(t)+\int_{0}^{t} \kappa(t, s, y(s)) \mathrm{d} s= & f(t), \\
& t \in I:=[0, T],
\end{aligned}
$$

has a unique solution. Then, the collocation solution $u_{h} \in$ $\left(\mathcal{S}_{m}^{(0)}\left(I_{h}\right)\right)^{r}$ to Eqn. (42) is defined by the equation

$$
A(t) u_{h}(t)+\int_{0}^{t} k\left(t, s, u_{h}(s)\right) \mathrm{d} s=f(t),
$$

for $t \in X_{h}$. Proceeding as in Section 3, we obtain the nonlinear equations

$$
\begin{aligned}
& f\left(t_{n, i}\right) \\
& =A\left(t_{n, i}\right) U_{n, i}+F_{n, i} \\
& \quad+h \int_{0}^{c_{i}} \kappa\left(t_{n, i}, t_{n}+s h_{n}, L_{0}(s) U_{n-1}\left(t_{n}\right)\right. \\
& \left.\quad+\sum_{j=1}^{m} L_{j}(s) U_{n, j}\right) \mathrm{d} s
\end{aligned}
$$

for $i=1, \ldots, m$, where the lag terms are defined by

$$
\begin{aligned}
& F_{n, i} \\
& =h \sum_{l=0}^{n-1} \int_{0}^{1} \kappa\left(t_{n, i}, t_{l}+s h_{l}, L_{0}(s) U_{l-1}\left(t_{n}\right)\right. \\
& \left.\quad+\sum_{j=1}^{m} L_{j}(s) U_{l, j}\right) \mathrm{d} s .
\end{aligned}
$$

A suitable method for solving this system is Newton's iterative method, since it can be proved (see Atkinson (2001)) that this method converges to the solution $U_{n, i}$ by the initial value $U_{n-1, i}$ for sufficiently small $h$. Subtracting (43) from (42), we obtain

$$
A(t) e(t)+\int_{0}^{t}\left(\kappa(t, s, y(s))-\kappa\left(t, s, u_{h}(s)\right)\right) \mathrm{d} s=0,
$$

for $t \in X_{h}$. Now supposing $k(t, s, y)$ is continuously differentiable with respect to $y$, using the mean value theorem and letting $t=t_{n, i}$, we obtain

$$
A\left(t_{n, i}\right) e\left(t_{n, i}\right)+\int_{0}^{t_{n, i}} \kappa_{y}\left(t_{n, i}, s, \eta(s)\right) e(s) \mathrm{d} s=0,
$$


where $\eta(s)$ is between $y(s)$ and $u(s)$. This equation is linear and its index can be considered an index for the nonlinear system (42). Hence for the nonlinear equation of the Hessenberg type

$$
\begin{aligned}
& {\left[\begin{array}{cccc}
A_{1,1}(t) & \ldots & A_{1, \nu-1}(t) & 0 \\
\vdots & \ddots & \vdots & \vdots \\
A_{\nu-1,1}(t) & \ldots & 0 & 0 \\
0 & \ldots & 0 & 0
\end{array}\right]\left[\begin{array}{c}
y_{1}(t) \\
y_{2}(t) \\
\vdots \\
y_{\nu}(t)
\end{array}\right]} \\
& +\int_{0}^{t}\left[\begin{array}{c}
k_{1}\left(t, s, y_{1}(s), y_{2}(s), \ldots, y_{\nu}(s)\right) \\
\vdots \\
k_{\nu-1}\left(t, s, y_{1}(s), y_{2}(s)\right) \\
k_{\nu}\left(t, s, y_{1}(s)\right)
\end{array}\right] \mathrm{d} s \\
& =\left[\begin{array}{c}
f_{1}(t) \\
f_{2}(t) \\
\vdots \\
f_{\nu}(t)
\end{array}\right]
\end{aligned}
$$

the index is $\nu$, if $\prod_{i=1}^{\nu} k_{i, y_{\nu+1-i}}\left(t, t, y_{1}(t), \ldots, y_{\nu+1-i}(t)\right)$ is invertible in a neighborhood of $\left(y_{1}(t), \ldots, y_{\nu}(t)\right)$. We should have the same results for the order of the collocation methods that we obtained in previous sections.

Theorem 5. Let

$$
\begin{gathered}
f(t) \in C^{m+2}\left(I, \mathbb{R}^{r}\right), \\
\frac{\partial^{i}}{\partial t^{i}} k\left(t, s, y_{1}(s), \ldots, y_{\nu+1-j}(s)\right) \in \mathbf{C}\left(D, \mathbb{R}^{r \times r}\right)
\end{gathered}
$$

for $i=1, \ldots, m+1$ and $j=1, \ldots, \nu$ and $A(t) \in$ $\mathbf{C}^{m+1}\left(I, \mathbb{R}^{r \times r}\right)$ be satisfied the system (45), and suppose that the system has Hessenberg form of index $\nu$. Also suppose that the consistency conditions of Theorem 1 hold for this system. Then the approximate solution $u_{h}$ of the (fully discretised) continuous collocation method with distinct collocation parameters $c_{1}, \ldots, c_{m} \in(0,1]$ (if it exists) converges to the solution $y$ if and only if

$$
\varrho=\max \left\{\left|\lambda_{1}\right|,\left|\lambda_{2}\right|\right\} \leq 1,
$$

where $\lambda_{1}$ and $\lambda_{2}$ are given by (18)-(22), and the collocation error satisfies

$$
\begin{gathered}
\left\|y_{1}-u_{1}\right\| \leq \begin{cases}\mathcal{O}\left(h^{m+1}\right) & \text { if } \lambda_{1}, \lambda_{2} \in[-1,1), \\
\mathcal{O}\left(h^{m}\right) & \text { if } \lambda_{1}=1 \text { or } \lambda_{2}=1,\end{cases} \\
\left\|y_{2}-u_{2}\right\| \leq \begin{cases}\mathcal{O}\left(h^{m}\right) & \text { if } \lambda_{1}, \lambda_{2} \in[-1,1), \\
\mathcal{O}\left(h^{m-2}\right) & \text { if } \lambda_{1}=1 \text { or } \lambda_{2}=1,\end{cases} \\
\vdots \\
\left\|y_{\nu}-u_{\nu}\right\| \leq \begin{cases}\mathcal{O}\left(h^{m+2-\nu}\right) & \text { if } \lambda_{1}, \lambda_{2} \in[-1,1), \\
\mathcal{O}\left(h^{m+2-2 \nu}\right) & \text { if } \lambda_{1}=1 \text { or } \lambda_{2}=1\end{cases}
\end{gathered}
$$

and hence

$\left\|y-u_{h}\right\| \leq \begin{cases}\mathcal{O}\left(h^{m+2-\nu}\right) & \text { if } \lambda_{1}, \lambda_{2} \in[-1,1) \\ \mathcal{O}\left(h^{m+2-2 \nu}\right) & \text { if } \lambda_{1}=1 \text { or } \lambda_{2}=1,\end{cases}$

where $u_{h}=\left[u_{1}, \ldots, u_{\nu}\right]^{T}$.

\section{Numerical experiments}

In this section, we illustrate the efficiency of the introduced methods by applying them to some linear and nonlinear problems, in comparison with the existing methods (e.g., Kauthen, 2001; Hadizadeh et al., 2011). In the designed package for these methods, we have provided techniques for solving linear and nonlinear systems. It is also worth mentioning that the capability of these methods for solving nonlinear and higher index problems distinguishes them from the methods mentioned above. We do the comparison after presenting some examples for confirming the theoretical results. We note that the order of the error is the slop of the function $\log (\|(e(h))\|)$ with respect to $\log (h)$ with $h=1 / N$. The numerical computations have been done using MATLAB and MAPLE.

Example 1. Let

$$
\begin{gathered}
A(t)=\left(\begin{array}{ccc}
\cos (t) & t+1 & 0 \\
5 & 0 & 0 \\
0 & 0 & 0
\end{array}\right), \\
k(t, s)=\left(\begin{array}{ccc}
-6 & t s-1 & 3 \\
\sin (t) & s+1 & 0 \\
\sin (t s)+1 & 0 & 0
\end{array}\right),
\end{gathered}
$$

and determine the function $f$ in such a way that the exact solution of (1) is

$$
y=\left(\begin{array}{c}
\sin (t) \\
\cos (t) \\
e^{t}
\end{array}\right) .
$$

For solving the system (1) on $[0,1]$, we use the FDCCM with $c=[0, .35, .8, .95]$. With this vector, $c$, we have $\lambda_{1}=-7.2578 \mathrm{e}-004$ and $\lambda_{2}=$ -0.8227 . Figure 1 shows logarithmic plots of three components of the error function with respect to $\log (h)=$ $-\log (N), N=2, \ldots, 40$, where the slopes of lines are $4,3,2$, respectively. These plots confirm the results of Theorem 3 .

In the proof of convergence properties of the perturbed continuous collocation methods for the SFVIE, we use interpolation of the error function with a smaller number of collocation parameters in the case $\lambda_{1}=1$ or $\lambda_{2}=1$. Hence, the order of the method may be greater than $m$. However, the existence of an example with 


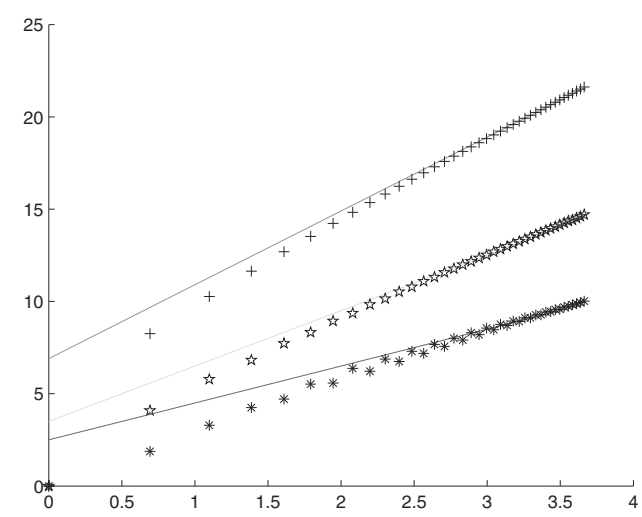

Fig. 1. Fully discretised continuous collocation methods with $c=[0, .35, .8, .95]$ in Example 1. The plotted function is $\log (\|e(h)\|)$ with respect to $\log (h)$. The symbols (plus, pentagon and star) are used to show the first, the second and the third components of the error function. The lines have respectively the slops 4,3 and 2 .

approximate solution of order $m$ shows that the obtained order is optimal. For the SFVIE, we refer the reader to the examples given by Kauthen and Brunner (1997). For IAEs, we give the following example.

Example 2. Let

$$
\begin{gathered}
A(t)=\left(\begin{array}{cc}
2+t & 0 \\
0 & 0
\end{array}\right), \\
k(t, s)=\left(\begin{array}{cc}
t & \exp (s) \\
\exp (-t s) & 0
\end{array}\right), \\
f=\left(\begin{array}{c}
-t+t \times \exp (t)+\sin (t)+(2+t) \exp (t) \\
\left(1-\exp \left(-t^{2}+t\right)\right) /(t-1)
\end{array}\right) .
\end{gathered}
$$

Then the exact solution of the index 2 IAE in (1) is given by

$$
y=\left(\begin{array}{c}
\exp (t) \\
\cos (t) \exp (-t)
\end{array}\right)
$$

For solving this IAE on $[0,1]$, we use FDCCMs with

$$
c=\left[0,0.3, .4, c_{5}, .95\right]
$$

and

$c_{3}$

$=0.8850192409343446350142328261033222536980988641879$.

With this vector, $c$, we have $\lambda_{1}=1$ and $\lambda_{2}=$ 0.0005727614821446677 . To obtain this collocation parameters, we use (18)-(20) to change the value of $c_{3}$ continuously until obtaining $\lambda=1$. We set "Digits:=50" in the MAPLE software to obtain more accurate results.

Tables 1 and 2 show the error of each component at the collocation points $t_{N, i}, i=1, \ldots, m$, for different values of $N$.
Table 1. Error of the continuous collocation method for Example 2 at the collocation points $t_{N, 1}, \ldots, t_{N, 4}$.

\begin{tabular}{|c|c|c|c|c|}
\hline$N$ & 16 & 32 & 64 & 128 \\
\hline \hline$\left\|e_{1}\left(t_{N, 1}\right)\right\|$ & $9.580 \mathrm{e}-12$ & $5.898 \mathrm{e}-13$ & $3.659 \mathrm{e}-14$ & $2.278 \mathrm{e}-15$ \\
\hline$\left\|e_{1}\left(t_{N, 2}\right)\right\|$ & $8.458 \mathrm{e}-12$ & $5.312 \mathrm{e}-13$ & $3.326 \mathrm{e}-14$ & $2.081 \mathrm{e}-15$ \\
\hline$\left\|e_{1}\left(t_{N, 3}\right)\right\|$ & $1.321 \mathrm{e}-11$ & $8.074 \mathrm{e}-13$ & $4.990 \mathrm{e}-14$ & $3.102 \mathrm{e}-15$ \\
\hline$\left\|e_{1}\left(t_{N, 4}\right)\right\|$ & $1.741 \mathrm{e}-11$ & $1.077 \mathrm{e}-12$ & $6.697 \mathrm{e}-14$ & $4.175 \mathrm{e}-15$ \\
\hline$\left\|e_{2}\left(t_{N, 1}\right)\right\|$ & $1.322 \mathrm{e}-7$ & $3.372 \mathrm{e}-8$ & $8.514 \mathrm{e}-9$ & $2.139 \mathrm{e}-9$ \\
\hline$\left\|e_{2}\left(t_{N, 2}\right)\right\|$ & $1.324 \mathrm{e}-7$ & $3.232 \mathrm{e}-8$ & $7.985 \mathrm{e}-9$ & $1.984 \mathrm{e}-9$ \\
\hline$\left\|e_{2}\left(t_{N, 3}\right)\right\|$ & $1.765 \mathrm{e}-7$ & $4.549 \mathrm{e}-8$ & $1.152 \mathrm{e}-8$ & $2.970 \mathrm{e}-9$ \\
\hline$\left\|e_{2}\left(t_{N, 4}\right)\right\|$ & $2.747 \mathrm{e}-7$ & $6.587 \mathrm{e}-8$ & $1.612 \mathrm{e}-8$ & $3.986 \mathrm{e}-9$ \\
\hline
\end{tabular}

Table 2. Order of the continuous collocation method for Example 2, where $p$ denotes the order.

\begin{tabular}{|c|c|c|c|c|c|}
\hline$N$ & 8 & 16 & 32 & 64 & 128 \\
\hline \hline$p\left(\left\|e_{1}\left(t_{N, 1}\right)\right\|\right)$ & 4.0890 & 4.0437 & 4.0217 & 4.0108 & 4.0054 \\
\hline$p\left(\left\|e_{1}\left(t_{N, 2}\right)\right\|\right)$ & 3.9649 & 3.9857 & 3.9935 & 3.9969 & 3.9985 \\
\hline$p\left(\left\|e_{1}\left(t_{N, 3}\right)\right\|\right)$ & 4.1452 & 4.0679 & 4.0328 & 4.0161 & 4.0080 \\
\hline$p\left(\left\|e_{1}\left(t_{N, 4}\right)\right\|\right)$ & 4.0544 & 4.0285 & 4.0145 & 4.0073 & 4.0037 \\
\hline$p\left(\left\|e_{2}\left(t_{N, 1}\right)\right\|\right)$ & 1.8685 & 1.9395 & 1.9710 & 1.9858 & 1.9929 \\
\hline$p\left(\left\|e_{2}\left(t_{N, 2}\right)\right\|\right)$ & 2.1237 & 2.0656 & 2.0338 & 2.0172 & 2.0087 \\
\hline$p\left(\left\|e_{2}\left(t_{N, 3}\right)\right\|\right)$ & 1.7907 & 1.9097 & 1.9577 & 1.9795 & 1.9899 \\
\hline$p\left(\left\|e_{2}\left(t_{N, 4}\right)\right\|\right)$ & 2.2144 & 2.1155 & 2.0603 & 2.0308 & 2.0156 \\
\hline
\end{tabular}

Remark 7. Tables 1 and 2 show that the order of the errors cannot be exceeded from what we proved in the case $\lambda_{1}=1$. These tables confirm the fact that the order of continuous collocation methods decreases by two when the index of the method increases by one for the special case $\lambda_{1}=1$.

Example 3. Let

$$
\begin{gathered}
A(t)=\left(\begin{array}{ll}
1 & 0 \\
0 & 0
\end{array}\right), \\
k_{1}\left(t, s, y_{1}, y_{2}\right)=\left(y_{1}^{2}+2\right) y_{2}+e_{2}^{y}, \\
k_{2}\left(t, s, y_{1}\right)=(1+s) y_{1}
\end{gathered}
$$

and

$$
=\left(\begin{array}{c}
e^{t}+\sin (t)-\frac{t \sin (2 t)}{4}+\frac{\sin (t)^{2}}{4}+\frac{5 t^{2}}{4}-1 \\
\sin (t)-\cos (t)-t \cos (t)+1
\end{array}\right) .
$$

Then the system (45) has the exact solution

$$
y=\left(\begin{array}{c}
\sin (t) \\
t
\end{array}\right) .
$$

For this system,

$$
\frac{\partial k_{1}}{\partial y_{2}} \frac{\partial k_{2}}{\partial y_{1}}=\left(\left(y_{1}^{2}+2\right)+e^{y_{2}}\right)(1+s)
$$


which is positive for all $y_{1}$ and $y_{2}$ and $s>-1$. Hence the index of this nonlinear system on $I:=[0,1]$ is 2 . For solving this IAE on $I$, we use FDCCMs with

$$
c=[0, .7, .9] .
$$

With this vector, $c$, we have $\lambda_{1}=0.8484$ and $\lambda_{2}=$ 0.0027 .

Table 3 shows the maximum error of each component at the collocation points and their convergence orders for different values of $N$. This table confirms the theoretical results for the nonlinear case (see Theorem 5).

7.1. Comparison. We chose the following examples by Hadizadeh et al. (2011) to make a comparison between the presented methods and Jacobi spectral methods of Hadizadeh et al. (2011).

Example 4. (Hadizadeh et al., 2011) Let

$$
\begin{gathered}
A(t)=\left(\begin{array}{ccc}
1 & 0 & 0 \\
0 & 1 & 0 \\
0 & 0 & 0
\end{array}\right), \\
k(t, s)=\left(\begin{array}{ccc}
-\frac{3-2 s}{2-s} & -\frac{3-s}{2-s} & -2(2-s) \\
-\frac{-1}{s-2} & +1 & -1 \\
-(s+2) & -\left(s^{2}-4\right) & 0
\end{array}\right),
\end{gathered}
$$

where $f$ is determined so that the exact solution of the index 2 IAE in (1), is

$$
y=\left(e^{t}, e^{t},-\frac{e^{t}}{2-t}\right)^{T}
$$

on $I=[0,1]$. Let $\left\{d_{i}\right\}_{i=1}^{\mathbf{N}}$ be the roots of the Chebyshev polynomial of degree $\mathbf{N}$. Then the collocation parameters proposed for FDCCMs are

$$
c=\left[0, \frac{1+d_{1}}{2}, \frac{1+d_{2}}{2}, \ldots, \frac{1+d_{\mathbf{N}}}{2}, 1\right],
$$

with $\lambda_{2}=(-1)^{\mathbf{N}+1}$, for $\mathbf{N}=1, \ldots, 12$. First, by setting $h=1$, we compare these methods with the Jacobi spectral method of degree $m+1$ (if the criteria of the comparison are the size of linear system it may be $m$ ). In Table 4 , we compare these results with the corresponding results of Hadizadeh et al. (2011), which shows the efficiency of the introduced methods.

Now, since the convergence results were only guaranteed for sufficiently small $h$, let us fix $m=7$ and compare those with the results of $h=1 / 2^{\mathcal{N}}, \mathcal{N}=$ $1, \ldots, 4$. Table 5 shows that these results are a little less efficient than the previous ones. Therefore, one may question, why we use the piecewise polynomials instead of one large degree polynomial as done by Hadizadeh et al. (2011). The answer is that, although the methods which use large degree polynomial in the whole interval of the solution are efficient for simple linear cases, they cannot be packaged for the nonlinear cases or stiff equations.

Example 5. (Hadizadeh et al., 2011) Let

$$
\begin{gathered}
A(t)=\left(\begin{array}{ll}
1 & 0 \\
0 & 0
\end{array}\right), \\
k(t, s)=\left(\begin{array}{cc}
-e^{t+s} & -(s+1)^{2} \\
-(s+t+2) & 0
\end{array}\right),
\end{gathered}
$$

where $f$ is determined so that the exact solution of the index 2 Hessenberg form in (1), is

$$
y=(\sin (t), \cos (2 t))^{T},
$$

on $I=[0,1]$. For this example we use the FDCCM with $c=[0,0.13,0.36,0.77,0.84,0.93,1], \lambda_{1}=0$ and $\lambda_{2}=0.05095$. Table 6 shows the results of this method for $\mathcal{N}=1,2,3,4$. This table shows that these methods are as efficient as those of Hadizadeh et al. (2011).

Table 6. Maximum absolute error of applying the FDCCM with $c=[0,0.13,0.36,0.77,0.84,0.93,1], m=7$ and different values of $h$ for Example 5 .

\begin{tabular}{|c|c|c|c|c|}
\hline $\mathcal{N}$ & $h$ & $c t$ & $\left\|e_{1}\right\|$ & $\left\|e_{2}\right\|$ \\
\hline \hline 1 & 1 & $0.003 \mathrm{~s}$ & $1.2255 \mathrm{e}-07$ & $4.4608 \mathrm{e}-05$ \\
\hline 2 & $1 / 2$ & $0.007 \mathrm{~s}$ & $1.0320 \mathrm{e}-09$ & $4.4916 \mathrm{e}-07$ \\
\hline 3 & $1 / 4$ & $0.015 \mathrm{~s}$ & $8.0568 \mathrm{e}-12$ & $3.4297 \mathrm{e}-09$ \\
\hline 4 & $1 / 8$ & $0.036 \mathrm{~s}$ & $7.8271 \mathrm{e}-14$ & $3.4259 \mathrm{e}-11$ \\
\hline
\end{tabular}

\section{Conclusion}

In this paper, we investigated integral algebraic equations of the Hessenberg type. We also stated a useful existence and uniqueness theorem for IAEs and analyzed the convergence properties of collocation methods and perturbed collocation methods for IAEs and SVIEs, respectively. The analysis showed that the order of collocation methods decreases by one when the index of methods increases by one for the case $\lambda_{1}<1$ or $\lambda_{2}<1$ and by two when one of the stability parameters $\lambda_{1}$ or $\lambda_{2}$ is equal to one. In the future it can be investigated if each IAE of index $\nu$ has the same convergence properties that we obtained for the Hessenberg type.

\section{References}

Atkinson, K. (2001). Theoretical Numerical Analysis: A Functional Analysis Framework, Springer-Verlag, New York, NY.

Bandrowski, B., Karczewska, A. and Rozmej, P. (2010). Numerical solutions to integral equations equivalent to differential equations with fractional time, International Journal of Applied Mathematics and Computer Science 20(2): 261-269, DOI: 10.2478/v10006-010-0019-1. 
Table 3. Maximum error of the continuous collocation method for Example 3 with different $N$ and their orders, where $p$ denotes the order.

\begin{tabular}{|c|c|c|c|c|}
\hline$N$ & $\left\|e_{1}\right\|$ & $p\left(\left\|e_{1}\right\|\right)$ & $\left\|e_{2}\right\|$ & $p\left(\left\|e_{2}\right\|\right)$ \\
\hline \hline 4 & 0.0011829778167 & - & 0.018220162160561 & - \\
\hline 8 & 0.0002323308386 & 2.3482 & 0.0116098006381 & 0.6502 \\
\hline 16 & 0.0000390187065 & 2.5739 & 0.0057849116304 & 1.0050 \\
\hline 32 & 0.0000056154572 & 2.7967 & 0.0021594336940 & 1.4216 \\
\hline 64 & 0.0000007480172 & 2.9083 & 0.0006760359897 & 1.6755 \\
\hline 128 & 0.0000000961413 & 2.9598 & 0.0001874215869 & 1.8508 \\
\hline 256 & 0.0000000121759 & 2.9811 & 0.0000489698659 & 1.9363 \\
\hline 512 & 0.0000000015317 & 2.9908 & 0.0000125030117 & 1.9696 \\
\hline 1024 & 0.0000000001918 & 2.9977 & 0.0000031864901 & 1.9722 \\
\hline
\end{tabular}

Table 4. Maximum absolute error of applying different FDCCMs for Example 4, with $h=1$ (i.e., $\left\|e_{i}\right\|$ for $i=1,2,3$ ) and the method of Hadizadeh et al. (2011) with the Jacobi spectral method of degree $m+1$ (i.e., $\left\|\widetilde{e}_{i}\right\|$ for $i=1,2,3$ ). The parameter $c t$ denotes the computation time of FDCCMs.

\begin{tabular}{|c|c|c|c|c|c|}
\hline $\mathbf{m}$ & 5 & 7 & 9 & 11 & 13 \\
\hline \hline$\lambda$ & -1 & $-1 \mathrm{~s}$ & -1 & -1 & -1 \\
\hline$c t$ & $0.010 \mathrm{~s}$ & $0.017 \mathrm{~s}$ & $0.025 \mathrm{~s}$ & $0.034 \mathrm{~s}$ & $0.045 \mathrm{~s}$ \\
\hline$\left\|e_{1}\right\|$ & $5.97 \mathrm{e}-05$ & $1.13 \mathrm{e}-07$ & $9.75 \mathrm{e}-11$ & $9.81 \mathrm{e}-14$ & $3.69 \mathrm{e}-14$ \\
\hline$\left\|\widetilde{e}_{1}\right\|$ & $3.12 \mathrm{e}-06$ & $7.66 \mathrm{e}-08$ & $1.99 \mathrm{e}-09$ & $5.33 \mathrm{e}-11$ & $1.37 \mathrm{e}-12$ \\
\hline$\left\|e_{2}\right\|$ & $2.43 \mathrm{e}-05$ & $6.05 \mathrm{e}-08$ & $5.15 \mathrm{e}-11$ & $4.97 \mathrm{e}-14$ & $3.55 \mathrm{e}-14$ \\
\hline$\left\|\widetilde{e}_{2}\right\|$ & $1.84 \mathrm{e}-06$ & $4.67 \mathrm{e}-08$ & $1.46 \mathrm{e}-09$ & $3.01 \mathrm{e}-11$ & $7.73 \mathrm{e}-13$ \\
\hline$\left\|e_{3}\right\|$ & $2.50 \mathrm{e}-03$ & $8.11 \mathrm{e}-06$ & $1.24 \mathrm{e}-08$ & $1.16 \mathrm{e}-11$ & $3.98 \mathrm{e}-11$ \\
\hline$\left\|\widetilde{e}_{3}\right\|$ & $1.30 \mathrm{e}-04$ & $4.61 \mathrm{e}-06$ & $1.59 \mathrm{e}-07$ & $5.38 \mathrm{e}-09$ & $1.74 \mathrm{e}-10$ \\
\hline
\end{tabular}

Table 5. Maximum absolute error of applying the FDCCM for Example 4, with $m=7$ and different values of $h$.

\begin{tabular}{|c|c|c|c|c|c|}
\hline $\mathcal{N}$ & $h$ & $c t$ & $\left\|e_{1}\right\|$ & $\left\|e_{2}\right\|$ & $\left\|e_{3}\right\|$ \\
\hline \hline 1 & 1 & $0.017 \mathrm{~s}$ & $1.1329 \mathrm{e}-07$ & $6.0452 \mathrm{e}-08$ & $8.1137 \mathrm{e}-06$ \\
\hline 2 & $1 / 2$ & $0.022 \mathrm{~s}$ & $4.5165 \mathrm{e}-10$ & $5.8178 \mathrm{e}-10$ & $1.1734 \mathrm{e}-07$ \\
\hline 3 & $1 / 4$ & $0.033 \mathrm{~s}$ & $1.0734 \mathrm{e}-12$ & $1.6924 \mathrm{e}-12$ & $1.5294 \mathrm{e}-09$ \\
\hline 4 & $1 / 8$ & $0.066 \mathrm{~s}$ & $4.0457 \mathrm{e}-13$ & $3.1708 \mathrm{e}-13$ & $9.5574 \mathrm{e}-10$ \\
\hline
\end{tabular}

Brunner, H. (1977). Discretization of Volterra integral equations of the first kind, Mathematics of Computation 31(139): 708-716.

Brunner, H. (1978). Discretization of Volterra integral equations of the first kind (II), Numerische Mathematik 30(2): 117-136.

Brunner, H. (2004). Collocation Methods for Volterra Integral and Related Functional Equations, Cambridge University Press, New York, NY.

Bulatov, M.V. (1994). Transformations of differential-algebraic systems of equations, Zhurnal Vychislitel'noi Matematiki $i$ Matematicheskoi Fiziki 34(3): 360-372.

Bulatov, M.V. (2002). Regularization of degenerate integro-differential equations, Computational Mathematics and Mathematical Physics 42(11): 1602-1608.

Chistyakov, V.F. (1987). On Singular Systems of Ordinary Differential Equations. Lyapunov Functions and Their Applications, Siberian Publishing House NAUKA, Novosibirsk, pp. 231-239.

Chistyakov, V.F. (1996). Algebro-Differential Operators With Finite-Dimensional Core, Siberian Publishing House NAUKA, Novosibirsk.
De Hoog, F.R. and Weiss, R. (1973a). High order methods for Volterra integral equations of the first kind, SIAM Journal on Numerical Analysis 10(4): 647-664.

De Hoog, F.R. and Weiss, R. (1973b). On the solution of Volterra integral equations of the first kind, Numerische Mathematik 21(1): 22-32.

Gear, C.W. (1990). Differential algebraic equations indices and integral algebraic equations, SIAM Journal on Numerical Analysis 27(6): 1527-1534.

Hadizadeh, M., Ghoreishi, F. and Pishbin, S. (2011). Jacobi spectral solution for integral algebraic equations of index-2, Applied Numerical Mathematics 61(1): 131-148.

Hochstadt, H. (1973). Integral Equations, John Wiley, New York, NY.

Kauthen, J.P. (1997). The numerical solution of Volterra integral-algebraic equations by collocation methods, Proceedings of the 15th IMACS World Congress on Scientific Computation, Modelling and Applied Mathematics, Berlin, Germany, Vol. 2, pp. 451-456.

Kauthen, J.P. (2001). The numerical solution of integral-algebraic equations of index 1 by polynomial spline collocation methods, Mathematics of Computation 70(236): 1503-1514. 
Kauthen, J.-P. and Brunner, H. (1997). Continuous collocation approximations to solutions of first kind Volterra equations, Mathematics of Computation 66(220): 1441-1459.

Lamm, P.K. (2005). Full convergence of sequential local regularization methods for Volterra inverse problems, Inverse Problems 21(3): 785-803.

Lamm, P.K. and Scofield, T.L. (2000). Sequential predictorcorrector methods for the variable regularization of Volterra inverse problems, Inverse Problems 16(2): 373-399.

Saeedi, H., Mollahasani, N., Mohseni Moghadam, M. and Chuev, G.N. (2011). An operational Haar wavelet method for solving fractional Volterra integral equations, International Journal of Applied Mathematics and Computer Science 21(3): 535-547, DOI: 10.2478/v10006-011-0042-x.

Weiss, R. (1972). Numerical Procedures for Volterra Integral Equations, Ph.D. thesis, Australian National University, Canberra.

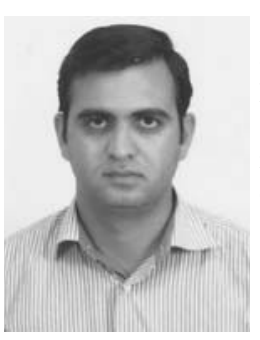

Babak Shiri is a Ph.D. student at the Faculty of Mathematical Sciences, University of Tabriz, Iran. He obtained his M.Sc. (2006) in numerical analysis from the same university. His research interests include integral-algebraic equations and differential-algebraic equations.

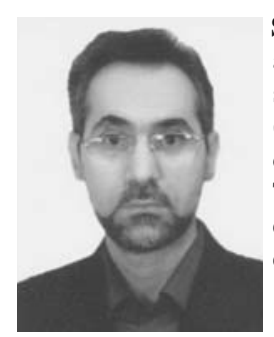

Sedaghat Shahmorad is an associate professor at the Faculty of Mathematical Sciences, University of Tabriz, Iran. He received his M.Sc. degree (1995) from the University of Tabriz and a Ph.D. degree (2002) from Tarbiat Modares University, Tehran, Iran, in mathematics and applied mathematics. His research interests include integral equations and integro-differential equations.

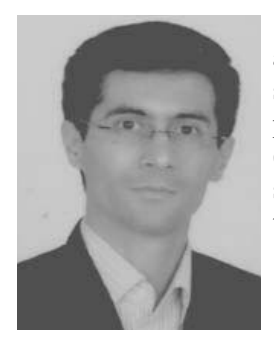

Gholamreza Hojjati is an associate professor at the Faculty of Mathematical Sciences, University of Tabriz, Iran. He received his Ph.D. in applied mathematics from the University of Tabriz (2005). His research interests include numerical solution of differential and Volterra integral equations, especially stiff systems.

\section{Appendix}

Lemma A1. Suppose that in the system (2), $A \in$ $\mathbf{C}^{\nu}\left(I, \mathbb{R}^{r \times r}\right), f \in \mathbf{C}^{\nu}\left(I, \mathbb{R}^{r}\right), k \in \mathbf{C}^{\nu}\left(D, \mathbb{R}^{r \times r}\right)$, $\prod_{i=1}^{\nu-1} A_{i, \nu-i}$ and $\prod_{i=1}^{\nu} k_{i, \nu+1-i}(t, t)$ are invertible, then the index of this system is $\nu$.

Proof. We prove the following statement by induction on $\nu$. There exist $A_{i}$ for $i=0, \ldots, \nu$, where the last $r_{1}$ columns of $A_{i}$ for $i=0, \ldots, \nu-1$ are zero column vectors and the 'rank degree' index of $A, k$, is $\nu$.

For $\nu=1$ this statement is true. We assume that the statement is true for $\nu=n$, and we show it is true for $\nu=n+1$. Set

$$
\begin{gathered}
A_{0}:=A=\left(\begin{array}{cc}
a & 0 \\
\Pi & 0
\end{array}\right), \\
k=\left(\begin{array}{cc}
\kappa & k_{1, n+1} \\
H & 0
\end{array}\right)
\end{gathered}
$$

with $a=\left[A_{1,1}, \ldots, A_{1, n}\right], \kappa=\left[k_{1,1}, \ldots, k_{1, n}\right]$,

$$
\Pi=\left[\begin{array}{cccc}
A_{2,1}(t) & \ldots & A_{2, n-1}(t) & 0 \\
\vdots & \ddots & \vdots & \vdots \\
A_{n, 1}(t) & \cdots & 0 & 0 \\
0 & \cdots & 0 & 0
\end{array}\right]
$$

and

$$
H=\left[\begin{array}{cccc}
k_{2,1}(t, s) & k_{2,2}(t, s) & \ldots & k_{2, n}(t, s) \\
\vdots & \vdots & \ddots & \vdots \\
k_{n, 1}(t, s) & k_{n, 2}(t, s) & \ldots & 0 \\
k_{n+1,1}(t, s) & 0 & \ldots & 0
\end{array}\right] .
$$

By the induction hypothesis, there exist $\Pi_{i}$ for $i=$ $0, \ldots, n$, where the last $r_{1}$ columns of $\Pi_{i}$ for $i=$ $0, \ldots, n-1$ are zero column vectors and the matrix $\Pi_{n}$ is invertible. Now we set

$$
\bar{B}_{0}=\left(\begin{array}{cc}
\alpha & B_{0} \\
0 & 0
\end{array}\right)
$$

with $\alpha=\left[0, \ldots, 0,\left(A_{1, n}^{-1}\right)^{T}\right]^{T}$, and we prove there exists a matrix $B_{0}$ of size $\left(r-r_{1}\right) \times\left(r-r_{1}\right)$ such that

$$
A_{0} \bar{B}_{0}=\left(\begin{array}{cc}
I & 0 \\
0 & \Pi_{0} \Pi_{0}^{-}
\end{array}\right),
$$

which implies $\bar{B}_{0}$ is a semi-inverse matrix of the matrix $A_{0}$. Therefore, the equations

$$
\Pi_{0} \alpha=0, \quad \Pi B_{0}=\Pi_{0} \Pi_{0}^{-}, a B_{0}=0
$$

should hold. Since the last $r_{1}$ columns of $\Pi_{0}$ are zero by hypothesis, the first equation holds. The equation $\Pi B_{0}=\Pi_{0} \Pi_{0}^{-}$makes $\left(r-r_{1}\right)\left(r-r_{1}\right)$ consistent equations (because $\Pi_{0}^{-}$is one of the solutions of this equations) and $\left(r_{1}\right) \times\left(r-r_{1}\right)$ unknowns of $\mathrm{B}$ are absence in this equation, and these unknowns can be found from $a B_{0}=0$.

Now, one can check that $A_{0}^{-}=\bar{B}_{0}$ and hence

$$
\begin{gathered}
I-A_{0} A_{0}^{-}=\left(\begin{array}{cc}
0 & 0 \\
0 & I-\Pi_{0} \Pi_{0}^{-}
\end{array}\right), \\
A_{1}=\left(\begin{array}{cc}
a & 0 \\
\Pi_{1} & 0
\end{array}\right), \quad k_{1}=\left(\begin{array}{cc}
\kappa & k_{1, n+1} \\
H_{1} & 0
\end{array}\right) .
\end{gathered}
$$


Using the hypothesis of induction, we proceed similarly to obtain

$$
A_{i}=\left(\begin{array}{cc}
a & 0 \\
\Pi_{i} & 0
\end{array}\right), \quad k_{i}=\left(\begin{array}{cc}
\kappa & k_{1, n+1} \\
H_{i} & 0
\end{array}\right)
$$

for $i=1, \ldots, \nu$.

Since the 'rank degree' index of $\Pi$ and $H$, is $\nu$, we use Lemma 2 to show that there exists invertible $A_{\nu+1}$, which completes the proof. To this end, we show that $\lambda A_{\nu}+k_{\nu}$ satisfy the 'rank degree' criterion

$$
\operatorname{rank} A_{\nu}=r-r_{1}
$$

and

$$
\begin{aligned}
& \operatorname{deg} \operatorname{det}\left(\lambda A_{\nu}+k_{\nu}\right) \\
& \quad=\operatorname{deg}(-1)^{n+2} \operatorname{det}\left(k_{1, n+1}\left(\lambda\left(\Pi_{i}\right)+H_{i}\right)\right) \\
& =\operatorname{deg}(-1)^{n+2} \lambda^{r-r_{1}} \operatorname{det}\left(k_{1, n+1} \Pi_{i}\right)=r-r_{1} .
\end{aligned}
$$

Received: 2 May 2012

Revised: 7 October 2012

Re-revised: 14 January 2013 